\title{
USO DE ICP OES E TITRIMETRIA PARA A DETERMINAÇÃO DE CÁLCIO, MAGNÉSIO E ALUMÍNIO EM AMOSTRAS DE SOLOS ${ }^{(1)}$
}

\author{
Ricardo Soares $^{(2)}$, Viviane Escaleira ${ }^{(3)}$, Maria Inês Couto Monteiro ${ }^{(4)}$, \\ Fernanda Veronesi Marinho Pontes ${ }^{(4)}$, Ricardo Erthal Santelli ${ }^{(2,6)}$ \& \\ Alberto Carlos de Campos Bernardi ${ }^{(5,6)}$
}

\begin{abstract}
RESUMO
O crescente interesse dos laboratórios brasileiros de análise de fertilidade de solos pela espectrometria de emissão óptica com plasma indutivamente acoplado (ICP OES) em detrimento da técnica de titrimetria deve-se à possibilidade da determinação simultânea de diferentes metais em uma ampla faixa de concentrações em extratos de interesse agronômico. Este estudo compara a precisão, reprodutibilidade e exatidão das duas técnicas de determinação de $\mathbf{C a}$, $\mathrm{Mg}$ e Al trocáveis extraídos com $\mathrm{KCl} 1 \mathrm{~mol} \mathrm{~L}{ }^{-1} \mathrm{em}$ amostras de solos. Foram analisadas 15 amostras de solos de referência, sendo três deles selecionados para avaliar a precisão e a reprodutibilidade das técnicas de determinação. A titrimetria e o ICP OES forneceram resultados mais reprodutíveis e precisos para a determinação de $\mathrm{Ca}, \mathrm{Mg}$ e $\mathrm{Al}$ quando o extrato foi analisado sem diluição. As técnicas de determinação diferiram estatisticamente $(p<0,05)$ pelo teste de identidade aplicado para comparação dos resultados de Ca, Mg e Al. Nas condições testadas, não se recomenda o uso de ICP OES para as determinações de Ca, Mg e Al trocáveis em amostras de solos.
\end{abstract}

Termos de indexação: ICP OES, titrimetria, cátions trocáveis e teste de identidade.

\footnotetext{
(1) Recebido para publicação em agosto de 2009 e aprovado em agosto de 2010.

(2) Pesquisadores do Departamento de Geoquímica, Universidade Federal Fluminense - UFF. Outeiro de São João Batista s/nº, CEP 24020-150 Niterói (RJ). E-mails: ricardosoaresuff@yahoo.com.br; santelli@geoq.uff.br

(3) Técnica da Embrapa Solos. Rua Jardim Botânico 1024, CEP 22460-000 Rio de Janeiro (RJ). E-mail: viviane@cnps.embrapa.br

(4) Pesquisadoras do Centro de Tecnologia Mineral - CETEM. Av. Pedro Calmon 900, Ilha da Cidade Universitária, CEP 21941908 Rio de Janeiro (RJ). E-mail: mmonteiro@cetem.gov.br

(5) Pesquisador da Embrapa Pecuária Sudeste. Rod. Washington Luiz, km 234, CEP 13560-970 São Carlos (SP). E-mail: alberto@cppse.embrapa.br

(6) Bolsista do CNPq.
} 


\title{
SUMMARY: USE OF ICP OES AND TITRIMETRY FOR CALCIUM, MAGNESIUM AND ALUMINUM DETERMINATION IN SOIL SAMPLES
}

\begin{abstract}
The interest of soil Laboratories in Brazil in inductively coupled plasma optical emission spectrometry (ICPOES) instead of titrimetry is on the rise due to the possibility of simultaneous determination of different metals within a wide range of concentrations in extracts of agronomic interest. This study compared: precision, reproducibility and accuracy of both techniques for exchangeable $\mathrm{Ca}, \mathrm{Mg}$ and $\mathrm{Al}$ determination extracted with $\mathrm{KCl} 1 \mathrm{~mol} \mathrm{~L}^{-1}$ from tropical soil samples. Fifteen reference soil samples were analyzed. Ca, $\mathrm{Mg}$ and Al concentrations determined by titrimetry and ICP OES were most reproducible and precise when the extracts were not diluted. The techniques differed statistically $(p<0.05)$ by the identity test applied to compare concentrations of $\mathrm{Ca}, \mathrm{Mg}$ and $\mathrm{Al}$. The determination of $\mathrm{Ca}, \mathrm{Mg}$ and $\mathrm{Al}$ in soil samples by ICP OES is not recommended under the tested conditions.
\end{abstract}

Index terms: ICP OES, titrimetry, exchangeable cations and identity test.

\section{INTRODUÇÃO}

A análise química do solo é uma das ferramentas mais utilizadas no Brasil para avaliação da fertilidade. Pelo uso das técnicas analíticas que envolvem extrações e determinações dos teores dos elementos químicos procura-se determinar o grau de suficiência ou deficiência destes no solo, além de quantificar condições adversas que possam prejudicar o desenvolvimento das plantas (Raij et al., 2001; Abreu et al., 2003; Soratto \& Crusciol, 2008).

Um programa de análise para avaliação e melhoramento da fertilidade do solo envolve as fases de amostragem, análise em laboratório, correlação dos resultados das análises com respostas à produção, interpretação e recomendações, transferência da informação aos produtores e pesquisa (Raij et al., 2001; Bernardi et al., 2002).

Atualmente, um dos maiores interesses nas análises de solos em laboratório é a seleção de técnicas de extração e de determinação desses elementos, os quais devem ser rápidos, exatos e de custo reduzido (Soltanpour et al., 1982; Valladares et al., 2001). Muitos trabalhos têm sido feitos no Brasil com esse intuito, porém os constantes aprimoramentos dos equipamentos e das técnicas analíticas fazem com que esse tema seja constantemente revisto e reavaliado (Coscione \& Raij, 1998; Raij et al., 2001; Valladares et al., 2001; Abreu et al., 2003; Boeira et al., 2004; Fonseca et al., 2005; Andrade et al., 2008).

$\mathrm{Na}$ avaliação da fertilidade do solo, os laboratórios brasileiros de análise utilizam amplamente a solução de $\mathrm{KCl} 1 \mathrm{~mol} \mathrm{~L}^{-1}$ para extração dos cátions trocáveis do solo: $\mathrm{Ca}^{2+}, \mathrm{Mg}^{2+}$ e, ou, $\mathrm{Al}^{3+}$ (Silva et al., 1998; Hislop \& Hornbeck, 2002; Bernardi et al., 2002; Abreu et al., 2003; Boeira et al., 2004; Soratto \& Crusciol, 2008). A maioria desses laboratórios determina $\mathrm{Ca}$ e $\mathrm{Mg}$ trocáveis por titrimetria de complexação com EDTA, na presença dos indicadores metalocrômicos calcon para Ca e negro de eriocromo $\mathrm{T}$ para $\mathrm{Ca}+\mathrm{Mg}$, sendo o Mg obtido por diferença (Silva et al., 1998; Bernardi et al., 2002). A determinação de Al trocável ocorre por meio de titrimetria de neutralização, utilizando hidróxido de sódio na presença do indicador azul de bromotimol (Silva et al., 1998; Bernardi et al., 2002; Abreu et al., 2003).

A literatura sugere que as determinações de $\mathrm{Ca}$, $\mathrm{Mg}$ e Al também possam ser feitas por espectrometria de emissão óptica com plasma indutivamente acoplado, ICP OES (Soltanpur et al., 1982; Raij et al., 2001; Fonseca et al., 2005), com as vantagens inerentes a essa técnica, como: análise simultânea, detecção em ampla faixa de concentração, alta precisão, exatidão, sensibilidade e rapidez. Devido às vantagens citadas do ICP OES, algumas instituições de pesquisa e laboratórios privados estão utilizando-o na determinação dos analitos em diferentes extratos de solos e plantas (Raij et al., 2001; Milagres et al., 2007). Entretanto, foi identificado efeito de matriz (EM) com supressão de sinal nas linhas de $\mathrm{Mg}$ devido ao aumento da concentração de KCl em solução (Budic \& Hudnik, 1994; Budic, 2000). Até o momento não foi realizado estudo de validação do método de determinação simultânea de $\mathrm{Ca}, \mathrm{Mg}$ e $\mathrm{Al}$ em extratos de $\mathrm{KCl}$ por ICP OES, em solos tropicais.

O objetivo deste estudo foi comparar as técnicas titrimétricas e ICP OES para a determinação de $\mathrm{Ca}$, $\mathrm{Mg}$ e $\mathrm{Al}$ em amostras de solos.

\section{MATERIAL E MÉTODOS}

Neste estudo, utilizaram-se 15 amostras de solos de referência oriundas do Programa de Análise da Qualidade de Laboratórios de Fertilidade (PAQLF). Os valores de referência foram determinados pelo método Embrapa (Bernardi et al., 2002) (Quadro 1). Nesse quadro também estão algumas características dessas amostras de solo, como granulometria, $\mathrm{pH}$ e capacidade de troca catiônica (CTC). 
Quadro 1. Análise granulométrica, pH, capacidade de troca catiônica (CTC), intervalo de confiança (IC), médias e desvios-padrão (s) de cálcio, magnésio e alumínio das amostras de referência do Programa de Análise da Qualidade de Laboratórios de Fertilidade

\begin{tabular}{|c|c|c|c|c|c|c|c|c|c|c|c|c|c|c|c|c|c|}
\hline \multirow{2}{*}{ Solo } & \multirow{2}{*}{ Argila } & \multirow{2}{*}{ Silte } & \multirow{2}{*}{ Areia } & \multirow{2}{*}{$\mathrm{pH}_{\mathrm{H}_{2} \mathrm{O}}$} & \multirow{2}{*}{ CTC } & \multicolumn{4}{|c|}{ Cálcio } & \multicolumn{4}{|c|}{ Magnésio } & \multicolumn{4}{|c|}{ Alumínio } \\
\hline & & & & & & \multicolumn{2}{|c|}{$-\mathrm{IC}-$} & \multirow[t]{2}{*}{ Média } & \multirow[t]{2}{*}{$\mathbf{s}$} & \multicolumn{3}{|c|}{ —IC - Média } & \multirow[t]{2}{*}{$\mathbf{s}$} & \multicolumn{2}{|c|}{$-\mathrm{IC}-$} & \multirow[t]{2}{*}{ Média } & \multirow[t]{2}{*}{$\mathbf{s}$} \\
\hline & & $\mathrm{gog}^{-1}$ & & & & & & & & $-\mathrm{cm}$ & ${ }^{o l} l_{c} \mathrm{dm}$ & & & & & & \\
\hline 1 & 100 & 76 & 824 & 4,5 & 4,73 & 0,30 & 0,60 & 0,43 & 0,09 & 0,10 & 0,30 & 0,20 & 0,07 & 0,70 & 1,40 & 1,00 & 0,20 \\
\hline 2 & 360 & 120 & 520 & 5,2 & 7,26 & 1,40 & 2,70 & 2,10 & 0,30 & 0,60 & 2,00 & 1,50 & 0,40 & 0,10 & 0,20 & 0,13 & 0,04 \\
\hline 3 & 480 & 316 & 204 & 5,6 & 21,82 & 8,00 & 13,20 & 10,50 & 1,40 & 3,20 & 6,90 & 5,00 & 0,90 & 0,10 & 0,20 & 0,15 & 0,04 \\
\hline 4 & 340 & 106 & 554 & 6,5 & 9,39 & 3,30 & 5,70 & 4,50 & 0,60 & 1,70 & 3,60 & 2,60 & 0,50 & 0,00 & 0,00 & 0,00 & 0,02 \\
\hline 5 & 180 & 132 & 688 & 4,6 & 7,72 & 0,30 & 0,60 & 0,47 & 0,08 & 0,20 & 0,50 & 0,30 & 0,10 & 1,10 & 1,70 & 1,40 & 0,20 \\
\hline 6 & 344 & 140 & 511 & 4,5 & 9,52 & 0,60 & 1,20 & 0,90 & 0,20 & 0,60 & 1,10 & 0,80 & 0,10 & 0,80 & 1,50 & 1,20 & 0,20 \\
\hline 7 & 318 & 179 & 503 & 6,1 & 14,89 & 4,60 & 7,40 & 6,00 & 0,70 & 2,20 & 4,70 & 3,50 & 0,60 & 0,00 & 0,00 & 0,01 & 0,04 \\
\hline 8 & 527 & 228 & 250 & 4,7 & 12,31 & 1,20 & 2,20 & 1,70 & 0,30 & 0,90 & 1,90 & 1,40 & 0,30 & 1,50 & 2,80 & 2,10 & 0,35 \\
\hline 9 & 151 & 93 & 753 & 6,0 & 6,88 & 2,20 & 3,50 & 2,90 & 0,30 & 0,90 & 1,90 & 1,40 & 0,20 & 0,00 & 0,10 & 0,05 & 0,03 \\
\hline 10 & 39 & 38 & 897 & 5,8 & 2,58 & 0,50 & 1,00 & 0,70 & 0,10 & 0,20 & 0,60 & 0,40 & 0,10 & 0,00 & 0,10 & 0,02 & 0,03 \\
\hline 11 & 241 & 118 & 635 & 5,0 & 7,15 & 1,70 & 2,60 & 2,10 & 0,20 & 0,50 & 1,20 & 0,90 & 0,20 & 0,10 & 0,30 & 0,20 & 0,07 \\
\hline 12 & 227 & 190 & 587 & 5,6 & 11,6 & 2,50 & 4,60 & 3,60 & 0,50 & 2,00 & 4,00 & 3,00 & 0,50 & 0,00 & 0,10 & 0,06 & 0,04 \\
\hline 13 & 241 & 118 & 636 & 5,1 & 7,61 & 1,20 & 2,30 & 1,70 & 0,40 & 1,00 & 1,80 & 1,40 & 0,30 & 0,10 & 0,30 & 0,20 & 0,07 \\
\hline 14 & 169 & 89 & 723 & 5,2 & 4,97 & 0,10 & 0,20 & 0,15 & 0,02 & 0,10 & 1,00 & 0,70 & 0,02 & 0,19 & 0,69 & 0,25 & 0,04 \\
\hline 15 & 740 & 97 & 163 & 3,7 & 1,44 & 0,10 & 0,70 & 0,40 & 0,05 & 0,10 & 0,70 & 0,30 & 0,05 & 0,18 & 0,70 & 0,30 & 0,05 \\
\hline
\end{tabular}

O procedimento de extração dos cátions trocáveis foi idêntico àquele descrito por Silva et al. (1998): adição de $100 \mathrm{~mL}$ de $\mathrm{KCl} 1 \mathrm{~mol} \mathrm{~L}^{-1}$ a $10 \mathrm{~cm}^{3}$ de amostra, agitação por 5 min, filtração e separação de quatro alíquotas de $25,00 \mathrm{~mL}$ do filtrado (extrato). Todas as extrações foram realizadas em triplicata.

A determinação de $\mathrm{Ca}$ foi feita por titrimetria, como se segue: à alíquota de $25 \mathrm{~mL}$ de extrato foram adicionados $5 \mathrm{~mL}$ de uma solução composta por: $200 \mathrm{~g}$ de $\mathrm{NaOH}, 5$ g de KCN, $100 \mathrm{~mL}$ de trietanolamina, cerca de $30 \mathrm{mg}$ de calcon e água suficiente para completar 1 L. Em seguida, Ca foi determinado por titulação, utilizando uma solução de EDTA $0,005 \mathrm{~mol} \mathrm{~L}^{-1}$.

A determinação de $\mathrm{Ca}+\mathrm{Mg}$ foi feita por titrimetria, da seguinte maneira: à alíquota de $25 \mathrm{~mL}$ de extrato foram adicionados $4 \mathrm{~mL}$ de solução-tampão $\mathrm{pH}=10$ composta de: $68 \mathrm{~g} \mathrm{NH}_{4} \mathrm{Cl}, 0,61 \mathrm{~g}$ de $\mathrm{NH}_{4} \mathrm{OH}, 0,8 \mathrm{~g}$ de $\mathrm{MgSO}_{4} \cdot 7 \mathrm{H}_{2} \mathrm{O}, 0,93 \mathrm{~g}$ EDTA-dissódico, $5 \mathrm{~g}$ de KCN, $100 \mathrm{~mL}$ de trietanolamina e água suficiente para completar $1 \mathrm{~L}$. Em seguida, $\mathrm{Ca}+\mathrm{Mg}$ foi determinado por titulação, utilizando uma solução de EDTA 0,005 mol Li-1 e indicador negro de eriocromo $\mathrm{T}$.

$\mathrm{O} \mathrm{Al}$ foi determinado na terceira alíquota por titrimetria de neutralização com $\mathrm{NaOH} 0,025 \mathrm{~mol} \mathrm{~L}^{-1}$, na presença de azul de bromotimol (Silva et al., 1998). A quarta alíquota foi subdividida em três. Uma subalíquota não foi diluída, enquanto as outras duas foram diluídas cinco e 10 vezes. Então, os cátions trocáveis $\mathrm{Ca}, \mathrm{Mg}$ e $\mathrm{Al}$ foram determinados por ICP OES. As diluições dos extratos salinos foram feitas para amenizar ou extinguir qualquer eventual interferência proveniente de efeito de matriz (EM) e avaliar a possibilidade de usar soluções menos salinas, ou seja, menos agressivas aos espectrômetros de emissão óptica (Nolte, 2003). Foi utilizado um equipamento da Perkin-Elmer, modelo OPTIMA 3000, e software ICP-Winlab.

As condições de trabalho do equipamento foram: $1.500 \mathrm{~W}$ de potência incidente, $15 \mathrm{~L} \mathrm{~min}^{-1}$ de vazão de gás argônio no plasma, $0,5 \mathrm{~L} \mathrm{~min}^{-1}$ de vazão de gás auxiliar, $0,80 \mathrm{~L} \mathrm{~min}^{-1}$ de vazão de nebulização, vazão de purga do gás normal, resolução normal, em triplicata, tempo automático de leitura (1 a 10 s), 2,0 $\mathrm{mL} \mathrm{min}^{-1}$ de taxa de vazão da amostra, 4,0 mL min ${ }^{-1}$ de taxa de fluxo de amostra, $55 \mathrm{~s}$ de intervalo de tempo de leitura. Os comprimentos de onda e as alturas de janelas otimizadas, utilizados respectivamente para cada um dos elementos em estudo, foram: $317,93 \mathrm{~nm}$ e $9,0 \mathrm{~mm}$ para Ca; $279,207 \mathrm{~nm}$ e 9,0 mm para $\mathrm{Mg}$; e 308,209 $\mathrm{nm}$ e 9,0 mm para Al.

Os limites de deteç̧ão (LD) e de quantificação (LQ) foram calculados pelas equações $\mathrm{LD}=3,3 \mathrm{~s} / \mathrm{b}$ e $\mathrm{LQ}=$ $10 \mathrm{~s} / \mathrm{b}$, em que (s) é o desvio-padrão de 10 determinações do branco e (b) é a inclinação da curva de calibração (Ribeiro et al., 2008).

Para determinação da precisão e reprodutibilidade, foram selecionadas três amostras de solos: 6,8 e 12, que apresentaram grande amplitude na variação dos teores dos elementos trocáveis. As amostras foram analisadas em triplicata, num total de 10 séries, no tempo de $24 \mathrm{~h}$. A precisão diz respeito à repetibilidade dentro de uma série de análises, enquanto a reprodutibilidade se refere à medida da aproximação dos resultados médios obtidos com o mesmo método e a mesma amostra sob diferentes séries de análises. A precisão foi representada pelo desvio-padrão relativo (DPR) calculado dentro da série, e a reprodutibilidade, 
pelo DPR calculado entre as diferentes séries (Milagres et al., 2007). A exatidão dos métodos foi determinada por comparação dos resultados obtidos para as 15 amostras de solo com os valores obtidos pelo PAQLF, utilizando a equação 1 (Ribeiro et al., 2008):

$$
\text { Exatidão }=(\mathrm{Xi} / \mathrm{Xv}) \times 100
$$

em que Xi é o valor médio das determinações para cada elemento em cada situação e Xv é o valor médio obtido pelo PAQLF.

Para comparação dos métodos de determinação, os tratamentos originaram-se do esquema fatorial $4 \mathrm{x}$ 15, dispostos em blocos casualizados completos com três repetições. Os fatores corresponderam aos quatro métodos (ICP OES e extrato sem diluição; ICP OES e extrato com diluição de cinco vezes; ICP OES com diluição de 10 vezes; e titrimetria) e às 15 amostras.

Foi aplicado também, para cada elemento, o teste de identidade estatística entre duas técnicas analíticas distintas, como proposto por Leite \& Oliveira (2002). Para testar as hipóteses $\mathrm{H}_{0}: \beta^{\prime}=\left[\begin{array}{ll}0 & 1\end{array}\right], \mathrm{H}_{0}: \bar{e}=0 \mathrm{e}$ avaliar o coeficiente de correlação linear $r$, o teste combina o teste $\mathrm{F}\left(\mathrm{H}_{0}\right)$, modificado por Graybill (1976), o teste do valor do erro médio tê , que indica se diferenças ocorrem ao acaso, e o coeficiente de correlação linear (r). Segundo esses autores, as técnicas analíticas serão estatisticamente iguais se, simultaneamente, após o ajuste da regressão linear, $\mathrm{Y}=\beta_{0}+\beta_{1} \mathrm{X}+\varepsilon, \beta_{0}$ for igual a 0 (coeficiente linear da equação ou intercepto) e $b_{1}$ for igual a 1 (coeficiente angular da equação ou coeficiente de regressão), estando o valor do coeficiente de correlação (r) próximo a 1. Com base nessas informações, elaborou-se regra decisória para testar a hipótese de identidade entre quaisquer pares de técnicas de determinação confrontadas (Quadro 2) (Leite \& Oliveira, 2002; Milagres et al., 2007).

Quadro 2. Regra de decisão para a comparação dos resultados obtidos por ICP OES e titrimetria nos extratos de $\mathrm{KCl}$ diluídos e não diluídos

\begin{tabular}{|c|c|c|c|c|}
\hline Situação & $\mathrm{F}\left(\mathrm{H}_{0}\right)$ & $\mathrm{t} \bar{e}$ & $\mathbf{r}$ & Decisão \\
\hline 1 & $\mathrm{~ns}$ & $\mathrm{~ns}$ & $\mathrm{r} \geq(1-|\bar{e}|)$ & ICP OES $(\mathrm{n})=$ Titrimetria \\
\hline 2 & $\mathrm{~ns}$ & $\mathrm{~ns}$ & $\mathrm{r} \leq(1-|\bar{e}|)$ & ICP OES $(\mathrm{n}) \neq$ Titrimetria \\
\hline 3 & $\mathrm{~ns}$ & * & $\mathrm{r} \geq(1-|\bar{e}|)$ & ICP OES $(\mathrm{n}) \neq$ Titrimetria \\
\hline 4 & $\mathrm{~ns}$ & * & $\mathrm{r} \geq(1-|\bar{e}|)$ & ICP OES $(\mathrm{n}) \neq$ Titrimetria \\
\hline 5 & * & $\mathrm{ns}$ & $\mathrm{r} \geq(1-|\bar{e}|)$ & ICP OES $(n) \neq$ Titrimetria \\
\hline 6 & * & $\mathrm{ns}$ & $\mathrm{r} \geq(1-|\bar{e}|)$ & ICP OES $(\mathrm{n}) \neq$ Titrimetria \\
\hline 7 & * & * & $\mathrm{r} \geq(1-|\bar{e}|)$ & ICP OES $(\mathrm{n}) \neq$ Titrimetria \\
\hline 8 & * & * & $\mathrm{r} \leq(1-|\bar{e}|)$ & ICP OES $(\mathrm{n}) \neq$ Titrimetria \\
\hline
\end{tabular}

ns e * denotam, respectivamente, não significativo e significativo a 5 \%. Fonte: Leite \& Oliveira (2002).

\section{RESULTADOS E DISCUSSÃO}

\section{Limites de detecção (LD) e quantificação (LQ)}

Para Ca e Mg, os LD e LQ obtidos no ICP OES foram cerca de quatro vezes menores do que aqueles obtidos pela titrimetria (Quadro 3). Por outro lado, para a determinação de Al por ICP OES, ambos os limites, LD e LQ, foram 10 vezes menores quando comparados aos obtidos pela titrimetria (Quadro 3). Esses resultados estão de acordo com a capacidade do ICP OES em determinar baixas concentrações de diferentes analitos de interesse (Soltanpour et al., 1982; Raij et al., 2001; Nolte, 2003). O LD, para o Al obtido no extrato sem diluição e utilizando o ICP OES, foi menor que aquele encontrado por Hislop \& Hornbeck (2002) em solos ácidos do hemisfério norte.

Quadro 3. Limites de detecção (LD) e limites de quantificação (LQ) dos teores de $\mathrm{Ca}, \mathrm{Mg}$ e $\mathrm{Al}$ obtidos pelas diferentes técnicas

\begin{tabular}{lccc}
\hline Técnica & Ca & Mg & Al \\
\cline { 2 - 3 } & & $\mathrm{cm} \mathrm{ol}_{\mathrm{c}} \mathrm{d} \mathrm{m}^{-3}$ & \\
& & $\mathrm{LD}$ & \\
Titrimetria & 0,20 & 0,20 & 0,10 \\
ICP-OES & 0,05 & 0,05 & 0,01 \\
& & $\mathrm{LQ}$ & \\
Titrimetria & 0,61 & 0,61 & 0,30 \\
ICP-OES & 0,15 & 0,15 & 0,03 \\
\hline
\end{tabular}

A capacidade de determinar concentrações mais baixas que as obtidas pela titrimetria pode acarretar diferenças estatísticas significativas entre os resultados obtidos pelas duas técnicas, visto que os valores médios dos valores de referência foram obtidos em sua grande maioria em laboratórios que utilizam a titrimetria em detrimento de técnicas espectroscópicas mais modernas, como AAS ou ICP OES (Bernardi et al., 2002).

\section{Precisão e reprodutibilidade das técnicas de determinação}

De acordo com Klesta \& Bartz (1996) e Millagres et al. (2007), os DPR dentro da série (precisão) podem variar entre 5 e $10 \%$, enquanto os DPR entre as diferentes séries (reprodutibilidade) devem situar-se abaixo de $20 \%$. A titrimetria apresentou boa precisão e reprodutibilidade, com exceção da determinação de $\mathrm{Al}$ no solo 8, em que o DPR foi maior que $10 \%$ na avaliação da precisão e maior que $20 \%$ na avaliação da reprodutibilidade (Quadro 4). Os resultados de $\mathrm{Ca}$, $\mathrm{Mg}$ e Al obtidos por ICP OES, nos extratos não diluídos, apresentaram boa precisão e reprodutibilidade para todas as amostras de solos. Os resultados de $\mathrm{Ca}$ 
e Mg obtidos por ICP OES, nos extratos diluídos cinco vezes, foram precisos e reprodutíveis; entretanto, para $\mathrm{Al}$ foi preciso e reprodutivo somente nos solos 6 e 12. Os resultados de $\mathrm{Ca}$ e $\mathrm{Al}$ obtidos por ICP OES, nos extratos diluídos 10 vezes, foram precisos e reprodutíveis para os solos 6 e 12 e precisos e reprodutíveis para Mg para os solos 6 e 8 (Quadro 4).

O uso das diluições em cinco e 10 vezes dos extratos com KCl $1 \mathrm{~mol} \mathrm{~L}^{-1}$ com o objetivo de atenuar eventuais interferências de efeito de matriz resultou no comprometimento da precisão e reprodutibilidade nas determinações dos três cátions por ICP OES, contrariando o que é amplamente divulgado na literatura (Hislop \& Hornbeck, 2000; Nolte, 2003).

\section{Exatidão}

Os valores percentuais de recuperação de $\mathrm{Ca}, \mathrm{Mg}$ e $\mathrm{Al}$ obtidos por titrimetria (Quadro 5) foram mais próximos dos valores médios dos valores de referência do PAQLF (Quadro 1); logo, mais exatos, do que os obtidos por ICP OES, independentemente da diluição do extrato utilizada (Quadro 5). A titrimetria apresentou recuperações que variaram de: 84 a $107 \%$ para o Ca, 85 a $110 \%$ para o $\mathrm{Mg}$ e 73 a $200 \%$ para o

Quadro 4. Média, precisão e reprodutibilidade das técnicas de determinação de Ca, Mg e Al de três solos (6, 8 e 12) extraídos com $\mathrm{KCl}$, em 10 séries analíticas, com três repetições por série

\begin{tabular}{|c|c|c|c|c|c|c|c|c|c|c|c|c|}
\hline & \multicolumn{3}{|c|}{ Titrimetria } & \multicolumn{2}{|c|}{ ICP-OES } & & \multicolumn{2}{|c|}{ ICP-OES } & \multirow{2}{*}{$\frac{(B)}{12}$} & \multicolumn{3}{|c|}{ ICP-OES (C) } \\
\hline & 6 & 8 & 12 & 6 & 8 & 12 & 6 & 8 & & 6 & 8 & 12 \\
\hline & & & & & & & & & & & & \\
\hline Média $\left(\mathrm{cmol}_{\mathrm{c}} \mathrm{dm}^{-3}\right)$ & 0,81 & 1,56 & 3,33 & 0,77 & 1,30 & 3,24 & 0,58 & 1,19 & 1,84 & 0,55 & 0,99 & 1,81 \\
\hline Precisão (\%) & 4,00 & 5,66 & 3,46 & 4,22 & 1,03 & 2,77 & 8,46 & 1,71 & 9,14 & 8,46 & 11,31 & 9,14 \\
\hline \multirow[t]{2}{*}{ Reprodutibilidade (\%) ${ }^{(2)}$} & 8,87 & 12,15 & 10,13 & 10,09 & 4,50 & 12,35 & 17,30 & 6,30 & 18,21 & 16,89 & 21,30 & 19,32 \\
\hline & & & & & & & & & & & & \\
\hline Média $\left(\mathrm{cmol}_{\mathrm{c}} \mathrm{dm}^{-3}\right)$ & 0,80 & 1,33 & 3,00 & 0,77 & 1,33 & 2,81 & 0,57 & 1,12 & 2,5 & 0,45 & 1,08 & 2,5 \\
\hline Precisão (\%) & 4,00 & 2,13 & 5,00 & 4,84 & 1,22 & 4,23 & 4,33 & 2,76 & 8,08 & 4,33 & 2,76 & 12,97 \\
\hline \multirow[t]{2}{*}{ Reprodutibilidade (\%) } & 13,45 & 5,11 & 11,30 & 7,00 & 3,12 & 5,65 & 12,27 & 5,78 & 19,14 & 7,57 & 10,13 & 23,80 \\
\hline & & & & & & & & & & & & \\
\hline Média $\left(\mathrm{cmol}_{\mathrm{c}} \mathrm{dm}^{-3}\right)$ & 0,97 & 1,74 & 0,06 & 1,05 & 1,76 & 0,05 & 0,53 & 0,07 & 0,00 & 0,50 & 0,07 & 0,00 \\
\hline Precisão (\%) & 5,97 & 34,64 & 0,00 & 7,31 & 8,05 & 9,62 & 7,84 & 15,30 & 0,00 & 7,86 & 18,30 & 0,00 \\
\hline Reprodutibilidade (\%) & 8,27 & 39,21 & 5,84 & 8,79 & 10,11 & 12,15 & 9,27 & 24,50 & 0,00 & 10,30 & 30,30 & 0,00 \\
\hline
\end{tabular}

(1) Precisão: desvio-padrão relativo dos resultados dentro de uma mesma série analítica. ${ }^{(2)}$ Reprodutibilidade: desvio-padrão relativo dos resultados entre as séries analíticas. (A) Extratos não diluídos. (B) Extratos diluídos cinco vezes. (C) Extratos diluídos 10 vezes.

Quadro 5. Exatidão das técnicas analíticas obtidas através da avaliação das recuperações percentuais dos teores de $\mathrm{Ca}, \mathrm{Mg}$ e $\mathrm{Al}$ nos 15 solos de referência do PAQLF

\begin{tabular}{|c|c|c|c|c|c|c|c|c|c|c|c|c|}
\hline \multirow{3}{*}{ Solo } & \multicolumn{4}{|c|}{$\mathbf{C a}$} & \multicolumn{4}{|c|}{ Mg } & \multicolumn{4}{|c|}{$\mathrm{Al}$} \\
\hline & \multirow[t]{2}{*}{ Titrimetria } & \multicolumn{3}{|c|}{ ICP-OES } & \multirow[t]{2}{*}{ Titrimetria } & \multicolumn{3}{|c|}{ ICP-OES } & \multirow[t]{2}{*}{ Titrimetria } & \multicolumn{3}{|c|}{ ICP-OES } \\
\hline & & (A) & (B) & (C) & & (A) & (B) & (C) & & (A) & (B) & (C) \\
\hline & & \multicolumn{11}{|c|}{ Recuperação (\%) } \\
\hline 1 & 98 & 81 & 77 & 81 & 85 & 76 & 40 & 40 & 73 & 85 & 51 & 46 \\
\hline 2 & 84 & 78 & 63 & 57 & 87 & 72 & 64 & 60 & 77 & 92 & 64 & 76 \\
\hline 3 & 87 & 83 & 69 & 66 & 94 & 79 & 19 & 17 & 113 & 93 & 67 & 66 \\
\hline 4 & 107 & 82 & 73 & 70 & 107 & 83 & 7 & 6 & 120 & 200 & 300 & 0 \\
\hline 5 & 91 & 81 & 38 & 40 & 90 & 79 & 66 & 67 & 86 & 88 & 71 & 71 \\
\hline 6 & 90 & 86 & 64 & 61 & 100 & 96 & 71 & 56 & 81 & 93 & 44 & 42 \\
\hline 7 & 92 & 80 & 73 & 66 & 98 & 82 & 26 & 25 & 200 & 120 & 53 & 51 \\
\hline 8 & 92 & 79 & 71 & 58 & 95 & 93 & 79 & 80 & 84 & 87 & 0 & 0 \\
\hline 9 & 88 & 76 & 71 & 79 & 100 & 76 & 65 & 71 & 90 & 80 & 0 & 0 \\
\hline 10 & 84 & 74 & 73 & 74 & 100 & 83 & 77 & 75 & 88 & 90 & 0 & 0 \\
\hline 11 & 97 & 85 & 65 & 73 & 97 & 85 & 68 & 77 & 91 & 88 & 0 & 0 \\
\hline 12 & 93 & 90 & 55 & 51 & 100 & 92 & 86 & 88 & 107 & 83 & 0 & 0 \\
\hline 13 & 88 & 87 & 63 & 62 & 98 & 82 & 68 & 55 & 100 & 85 & 0 & 0 \\
\hline 14 & 103 & 80 & 73 & 73 & 104 & 84 & 68 & 63 & 104 & 78 & 0 & 0 \\
\hline 15 & 87 & 79 & 62 & 73 & 110 & 90 & 40 & 50 & 93 & 77 & 157 & 176 \\
\hline
\end{tabular}

(A) Extratos não diluídos. (B) Extratos diluídos cinco vezes. (C) Extratos diluídos 10 vezes. 
Al. Por sua vez, o ICP OES apresentou recuperações que variaram de: 38 a $90 \%$ para o $\mathrm{Ca}, 6$ a $96 \%$ para o $\mathrm{Mg}$ e 0 a $300 \%$ para o $\mathrm{Al}$.

Por um lado, todos os valores obtidos por titrimetria estiveram dentro dos intervalos de confiança dos materiais de referência do PAQLF (Quadro 1). Por outro, nem todos os valores obtidos por ICP OES mostraram comportamento semelhante ao citado anteriormente, podendo isso ser explicado pelo efeito deletério da interferência não espectroscópica proporcionada por uma matriz altamente salina (Budic, 2000). O uso dos extratos diluídos comprometeu as exatidões nas determinações de $\mathrm{Ca}$, $\mathrm{Mg}$ e $\mathrm{Al}$ por ICP OES.

\section{Comparação das técnicas de determinação}

Foram consideradas todas as 180 determinações possíveis (12 x 15), não havendo necessidade de descartar amostras com concentrações extremamente altas ou baixas. Pode-se com isso avaliar o comportamento das técnicas de determinação de $\mathrm{Ca}$, $\mathrm{Mg}$ e Al encontradas em solos tropicais aplicando-se o teste de identidade entre as técnicas analíticas (Leite \& Oliveira, 2002).

Primeiramente, na determinação de $\mathrm{Ca}$ em extratos não diluído e diluído em cinco vezes, o ICP OES, em relação à titrimetria, não apresentou erros sistemáticos $\left(\beta_{0}=0\right)$ nem erros proporcionais. Isso ocorreu porque as sensibilidades obtidas foram altas e não foram significativamente diferentes $\left(\beta_{1}=1\right) \mathrm{e}$ também apresentaram altas correlações, porém as diferenças entre as técnicas de determinação não ocorreram casualmente $\left(t_{\bar{e}}>t_{\alpha}(n-1)\right)$. Entretanto, usando os extratos diluídos 10 vezes, o ICP OES, em relação à titrimetria, apresentou erros sistemáticos, e as determinações também não ocorreram ao acaso.
Portanto, pode-se afirmar que em todas as determinações de $\mathrm{Ca}$ por ICP OES, em relação à titrimetria, a diferença entre as técnicas analíticas não ocorreu ao acaso (Quadro 6).

Para o $\mathrm{Mg}$, só não foram encontrados erros sistemáticos na determinação por ICP OES nos extratos diluídos cinco vezes, em relação à titrimetria. Adicionalmente, a determinação obtida por diluição em 10 vezes foi a única que não acarretou erros proporcionais na determinação do Mg por ICP OES. Assim, como visto para o Ca anteriormente, as diferenças entre ICP OES e a titrimetria não foram casuais (Quadro 6).

Embora não tenham sido encontrados erros sistemáticos nem erros proporcionais para as determinações de $\mathrm{Al}$, foram observadas diferenças tendenciosas $(r<0,900)$ entre as duas técnicas analíticas quando se diluíram os extratos (Quadro 6).

Embora o ICP OES possibilite as determinações simultâneas dos três cátions, ainda assim não foi capaz de se equiparar à técnica de titrimetria, possivelmente devido aos efeitos ocasionados por interferências não espectroscópicas proporcionados pelo forte efeito de matriz da solução extratora (Budic, 2000). Por sua vez, o uso de diferentes diluições dos extratos não possibilitou eliminar ou amenizar o efeito de matriz.

Para que possam ser amenizadas ou eliminadas as interferências não espectroscópicas da matriz e, consequentemente, seja feita a determinação de $\mathrm{Ca}$, $\mathrm{Mg}$ e Al por ICP OES em amostras de solos, sugere-se que estudos posteriores avaliem a utilização de outros recursos, como: correção de matriz, uso de padrão interno, calibração por adição de padrão, adição de surfactantes, separação de matriz e correção com modelos matemáticos (Hislop \& Hornbeck, 2000; Nolte, 2003).

\section{Quadro 6. Resultados do teste de identidade aplicado para comparação das técnicas analíticas}

\begin{tabular}{|c|c|c|c|c|c|c|c|c|c|}
\hline Elemento & $\mathrm{n}^{(1)}$ & $\mathrm{B}_{0}{ }^{(2)}$ & $\mathbf{B}_{1}^{(3)}$ & $\mathbf{r}^{(4)}$ & $\bar{e}^{(5)}$ & $\mathrm{F}\left(\mathrm{H}_{0}\right)^{(6)}$ & $\mathrm{t} \bar{e}^{(7)}$ & $\mathbf{r} \geq(1-|\bar{e}|)$ & Conclusão \\
\hline & & & & \multicolumn{2}{|c|}{ ICP OES } & \multicolumn{2}{|c|}{ (A) $\mathrm{x}$ Titrimetria } & & \\
\hline $\mathrm{Ca}$ & 45 & $0,13^{\mathrm{ns}}$ & $0,639^{\text {ns }}$ & 0,999 & $-0,3050$ & $4367,60^{*}$ & $41,83^{*}$ & $\operatorname{sim}$ & ICP OES $\neq$ Titrimetria \\
\hline $\mathrm{Mg}$ & 45 & $-0,14$ * & $0,676^{\mathrm{ns}}$ & 0,997 & $-0,4838$ & $1392,22^{*}$ & $19,01^{*}$ & $\operatorname{sim}$ & ICP OES $\neq$ Titrimetria \\
\hline \multirow[t]{2}{*}{$\mathrm{Al}$} & 45 & $\cdot 0,04^{\mathrm{ns}}$ & $0,808^{\mathrm{ns}}$ & 0,998 & $-0,4377$ & $375,10 \mathrm{~ns}$ & $10,00 *$ & $\operatorname{sim}$ & ICP OES $\neq$ Titrimetria \\
\hline & & & & \multicolumn{2}{|c|}{ ICP OES } & \multicolumn{2}{|c|}{ (B) $\mathrm{x}$ Titrimetria } & & \\
\hline $\mathrm{Ca}$ & 45 & $0,19^{\text {ns }}$ & $0,728^{\mathrm{ns}}$ & 0,996 & $-0,1659$ & $487,88^{*}$ & $9,13^{*}$ & $\operatorname{sim}$ & ICP OES $\neq$ Titrimetria \\
\hline $\mathrm{Mg}$ & 45 & $-0,11^{\mathrm{ns}}$ & $0,748^{\mathrm{ns}}$ & 0,985 & $-0,3858$ & $319,51^{*}$ & $11,19^{*}$ & $\operatorname{sim}$ & ICP OES $\neq$ Titrimetria \\
\hline \multirow[t]{2}{*}{$\mathrm{Al}$} & 45 & $0,00^{\mathrm{ns}}$ & $0,483^{\text {ns }}$ & 0,885 & $-0,4097$ & $91,08^{*}$ & $8,91^{*}$ & sim & ICP OES $\neq$ Titrimetria \\
\hline & & & & \multicolumn{2}{|c|}{ ICP OES } & \multicolumn{2}{|c|}{ (C) $\mathrm{x}$ Titrimetria } & & \\
\hline $\mathrm{Ca}$ & 45 & 0,08 * & $0,769^{\mathrm{ns}}$ & 0,990 & $-0,1936$ & $140,34^{*}$ & $7,93^{*}$ & $\operatorname{sim}$ & ICP OES $\neq$ Titrimetria \\
\hline $\mathrm{Mg}$ & 45 & 0,16 * & 0,547 * & 0,784 & $-0,2779$ & $48,89^{*}$ & $3,21^{*}$ & $\operatorname{sim}$ & ICP OES $\neq$ Titrimetria \\
\hline $\mathrm{Al}$ & 45 & $0,00^{\mathrm{ns}}$ & $0,448^{\mathrm{ns}}$ & 0,876 & $-0,4739$ & $101,51^{*}$ & $9,17^{*}$ & sim & ICP OES $\neq$ Titrimetria \\
\hline
\end{tabular}

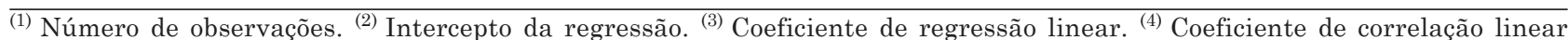
simples. ${ }^{(5)}$ Erro médio. ${ }^{\left({ }^{6}\right)}$ Teste $\mathrm{F}\left(\mathrm{H}_{0}\right)$. ${ }^{(7)}$ Teste t para o erro médio. ${ }^{\text {ns }} \mathrm{e}{ }^{* *}$ : não significativo e significativo a $5 \%$, respectivamente. (A) Extratos não diluídos. (B) Extratos diluídos cinco vezes. (C) Extratos diluídos 10 vezes. 


\section{CONCLUSÕES}

1. A técnica analítica ICP OES foi estatisticamente diferente da técnica titrimétrica na determinação de $\mathrm{Ca}, \mathrm{Mg}$ e Al. Nas condições testadas não se recomenda o uso do ICP OES para as determinações de $\mathrm{Ca}, \mathrm{Mg}$ e Al trocáveis extraídos com $\mathrm{KCl} 1 \mathrm{~mol} \mathrm{~L}^{-1} \mathrm{em}$ amostras de solos.

2. As diluições dos extratos não foram capazes de atenuar o efeito de matriz e, além disso, proporcionaram decréscimo nas precisões, reprodutibilidades e exatidões da técnica de ICP OES.

\section{AGRADECIMENTOS}

À Fundação Carlos Chagas Filho de Amparo à Pesquisa do Estado do Rio de Janeiro (FAPERJ), pela bolsa de doutorado do primeiro autor. Ao Conselho Nacional de Desenvolvimento Científico e Tecnológico (CNPq), pelas bolsas de produtividades. Ao Doutor Hélio Garcia Leite, por gentilmente ter cedido a planilha de confecção do teste de identidade.

\section{LITERATURA CITADA}

ABREU, C.H.; MURAOKA, T. \& LAVORANTE, A.F. Exchangeable aluminum evaluation in acid soils. Sci. Agric., 60:543-548, 2003.

ANDRADE, J.C.; COSCIONE, A.R.; POPPI, R.J. \& MELLO, C. Elimination of iron interference in the molecular spectrophotometric determination of aluminum in soil extracts using artificial neural networks. Anal. Sci., 24:1147-1150, 2008

BERNARDI, A.C.C.; SILVA, C.A.; PÉREZ, D.V. \& MENEGUELLI, N.A. Analytical quality program of soil fertility laboratories that adopt Embrapa methods in Brazil. Comm. Soil Sci. Plant Anal., 33:2661-2672, 2002.

BOEIRA, R. C.; RAIJ, B.van; SILVA, A.S. \& MAXIMILIANO, V.C.B. Extração simultânea de alumínio, cálcio, magnésio, potássio e sódio do solo com solução de cloreto de amônio. R. Bras. Ci. Solo, 28:29-936, 2004.

BUDIC, B. Radially resolved measurements of the emission intensity in the presence of matrix elements in inductively coupled plasma atomic emission spectrometry. Acta Chim. Slov., 47:153-163, 2000.

BUDIC, B. \& HUDNIK, V. Matrix effects of potassium chloride and phosphoric acid in argon inductively coupled plasma atomic emission spectrometry. J. Anal. Atm. Spectrum., 9:53-57, 1994.

COSCIONE, A.R. \& RAIJ, B.van. Revisiting titration procedures for the determination of exchangeable acidity and exchangeable aluminum in soils. Comm. Soil Sci. Plant Anal., 29:1973-1982, 1998
FONSECA, A.F.; ALLEONI, L.R.F.; MELFI, A.J. \& MONTES, C.R. Cation exchange capacity of an oxisol amended with an effluent from domestic sewage treatment. Sci. Agric., 6:552-558, 2005.

GRAYBILL, F.A. Theory and application of the linear model. Belmont, Duxbury Press, 1976. 704p.

HISLOP, J.E. \& HORNBECK, J.W. Coping with effects of high dissolved salt samples on the inductively coupled plasma spectrometer. Comm. Soil Sci. Plant Anal., 33:3377-3388, 2002

KLESTA, E. J. \& BARTZ, J. K. Quality assurance and control. In: BARTELS, J.M. \& BIGHAM, J.M., eds. Methods of soil analysis. Chemical methods. Madison, Soil Science Society of America, 1996. Part 3. 248p.

LEITE, H.G. \& OLIVEIRA, F.H.T. Statistical procedure to test identity between analytical methods. Comm. Soil Sci. Plant Anal., 33:1105-1118, 2002.

MILAGRES, J.J.M.; ALAVAREZ V., V.H.; CANTARUTTI, R.B. \& NEVES, J.C.L. Determinação de $\mathrm{Fe}, \mathrm{Zn}, \mathrm{Cu}$ e $\mathrm{Mn}$ extraídos do solo por diferentes extratores e dosados por espectrofotometria de emissão ótica em plasma induzido e espectrofotometria de absorção atômica. R. Bras. Ci. Solo, 31:237-245, 2007.

NOLTE, J. ICP: Emission spectrometry, A pratical guide Weinheim, Willey-VCH, 2003. 267p.

RAIJ, B.van; ANDRADE, J.C.; CANTARELLA, H. \& QUAGGIO, J.A. Análise química para avaliação da fertilidade de solos tropicais. Campinas, Instituto Agronômico, 2001. 285p.

RIBEIRO, F.A.L.; FERREIRA, M.M.C.; MORANO, S.C.; SILVA, L.R. \& SCHNEIDER, R.P. Planilha de validação: Uma nova ferramenta para estimar figuras de mérito na validação de métodos analíticos univariados. Química Nova, 31:164-171, 2008.

SILVA, F.C.; EIRA, P.A.; BARRETO, W.O.; PÉREZ, D.V. \& SILVA, C.A. Manual de métodos de análises químicas para avaliação da fertilidade do solo. Rio de Janeiro, Embrapa-CNPS, 1998. 56p. (Embrapa-CNPS. Documentos, 3).

SOLTANPOUR, P.N.; JONES JR., J.B. \& WORKMAN, S.M. Optical emission spectrometry. In: PAGE, A.L.; MILLER, R.H. \& KEENEY, D.R., eds. Methods of soil analysis. Chemical and microbiological properties. 2.ed. Madison, Soil Science Society of America, 1982. Part 2. p.29-65.

SORATTO, R.P. \& CRUSCIOL, C.A.C. Métodos de determinação de cálcio e magnésio trocáveis e estimativa do calcário residual em um Latossolo submetido a aplicação de calcário e gesso em superfície. R. Bras. Ci. Solo, 32:663-673, 2008.

VALLADARES, G.S.; PEREIRA, M.G.; SOUZA, J.M.P.; PÉREZ, D.V. \& ANJOS, L.H.C. Comparação entre os teores de nutrientes extraídos por três métodos em amostras de solos do Rio de Janeiro. R. Bras. Agroci., 7:137-141, 2001. 